[19] Vargas, F., Boyer, A., Bui, H. N., Salmi, L. R., Guenard, H., Gruson, D., Hilbert, G. (2008). Respiratory failure in chronic obstructive pulmonary disease after extubation: value of expiratory flow limitation and airway occlusion pressure after 0.1 second (P0.1). Journal of Critical Care, 23 (4), 577-584. doi: $10.1016 /$ j.jcrc.2007.12.009

[20] Kim, N.-S., Seo, J.-H., Ko, M.-H., Park, S.-H., Kang, S.-W., Won, Y. H. (2017). Respiratory Muscle Strength in Patients With Chronic Obstructive Pulmonary Disease. Annals of Rehabilitation Medicine, 41 (4), 659-666. doi: 10.5535/arm.2017.41.4.659

\title{
ELIMINATION OF CONGENITAL SYPHILIS IN UKRAINE: ANALYSIS AND THE EMERGING ISSUES
}

\author{
Lyudmyla Derevyanko \\ Department of preventive activities and coordination of co-operation with WHO \\ Ukrainian Institution of Strategic Studies of the Ministry of Health \\ 3 Volgo-Donsky ave., Kyiv, Ukraine, 02000 \\ l.derevyanko@kmu.edu.ua
}

\begin{abstract}
The objective of this study is to analyze the indicators of the process of elimination of mother-to-child transmission of syphilis in Ukraine thus helping to prepare for elimination of this process according to the WHO targets and criteria.

Epidemiological and clinical data of 237 women who had syphilis before or during pregnancy and babies born to them (238) from 1999 to 2007 years were presented. In addition were used statistical forms of the Public Health Center and Center for Medical Statistics of the Ministry of Health of Ukraine for the incidence of syphilis and HIV in women of reproductive age, pregnant women and children born to them, including those who were diagnosed with congenital syphilis (CS) and HIV infection.

Analysis of the causes of CS suggests that the main risk factors in Ukraine were the lack of prenatal care in $44 \%$ and treatment in $99 \%$ of pregnant women with syphilis. Besides out-of-date normative base regulating STI diagnostic and care create delay in diagnostics and treatment of pregnant women and their newborns.

In addition, the delay with prenatal diagnosis and treatment, late infection, quality of diagnostics, iatrogenic errors, refusal of women from current requirement for hospitalization, reinfection, and birth at home can be attributed to risk factors for CS.

Despite the main target indicator of CS elimination has been achieved, process indicators of syphilis as coverage of syphilis testing and treatment of pregnant women as well as other additional requirements have not reached yet.

On the way to the CS elimination Ukraine is facing a few challenges. There is an urgent need on further year the new strategy on STI/CS prevention with targets and targets indicators. In addition national protocols of the treatment of pregnant women should be adapted according to the European Guidelines as well as comprehensive data is required for surveillance and monitoring of CS elimination.
\end{abstract}

Keywords: congenital syphilis, HIV, mother-to-child transmission, elimination.

\section{Introduction}

For over a decade, WHO has adopted a numbers of policy documents on the global elimination of CS and new cases of HIV infection in infants and young children [1].

In 2007 WHO launched an initiative for the global elimination of congenital syphilis, outlined in "The global elimination of congenital syphilis: rationale and strategy for action" [2].

The global community has committed itself to eliminating mother-to-child transmission (MTCT) of HIV and syphilis as a public health priority [3].

Elimination of MTCT of HIV and syphilis is motivated by the fact that such a double elimination will facilitate the improvement of a wide range of results on the protection of the health of mothers and children. This integrated approach is necessary to improve the efficiency and quality of mother and child health $(\mathrm{MCH})$ services and to offer women more comprehensive primary care services. 
The term "congenital syphilis" has traditionally been used to describe the adverse outcomes of syphilis infection in pregnancy. However, expert consensus from a global consultation in 2012 suggested that, whenever possible, the term "mother-to-child transmission of syphilis" should be used in place of "congenital syphilis" in order to increase awareness of the full spectrum of adverse outcomes, including stillbirths, neonatal deaths, premature and low birth weight infants, as well as deformities at birth [4]. Investment in the elimination of MTCT of syphilis will contribute significantly to improved maternal and child health around the world, including achievement of MDGs 4, 5 and 6 , while also strengthening underlying health systems.

While WHO has announced the elimination of the transmission of HIV and syphilis in Cuba, Thailand, Belarus, Moldova and Armenia, Ukraine, like many other countries, has not achieved those goals yet [5].

\section{Aim of the research}

The objective of this study is to analyze the indicators of the process of elimination mother-tochild transmission of syphilis in Ukraine thus helping to prepare for validation of this process according to the WHO targets and criteria.

\section{Materials and methods.}

In retrospective study were presented epidemiological and clinical data of 237 women who had syphilis before or during pregnancy and babies born to them (238) from 1999 to 2007 years.

The first part included 163 mothers and their 164 babies that were on examination and treatment with suspected diagnosis of CS in the children city hospital of Kyiv. The second - data on 74 babies and their mothers obtained from the so called typical temporary cards of cases of CS (TTCCS), which were received from the city of Kyiv and 14 regions of Ukraine.

We used the national case definition for validation purposes that is consistent with the global surveillance definition [6]. The global surveillance case definition for CS is defined as:

- a stillbirth, live birth, or fetal loss at $>20$ weeks of gestation or $>500$ grams to a syphilis seropositive mother without adequate syphilis treatment;

- a stillbirth, live birth, or child aged $<2$ years with microbiological evidence of syphilis infection.

In addition, were used statistical forms of the Public Health Center (PHC) and Center for Medical Statistics (CMS) of the Ministry of Health of Ukraine (MHU) for the incidence of syphilis and HIV in women of reproductive age, pregnant women and children born to them, including those who were diagnosed with CS and HIV infection [7].

\section{Results}

We divided mothers who gave a birth to the newborn babies with suspected CS into three groups: group I - 102 mothers whose babies were diagnosed with the CS; group II - 78 mothers, who babies received so called prophylactic treatment; group III - 52 mothers whose babies have had a follow up at the Kyiv City Dermato-venereological Hospital. In further, for convenience, we will name the first group - CS group, the second - the PT group, the third - FU group.

The average age of mothers from CS group was $25.8 \pm 0.6$ years, $19.6 \%$ from them were non-residents of the city or town in which they delivered, there were more unmarried women (57 \%) compare with married or those living in civil marriage (36\%).

Only $21.5 \%$ of women were at risk groups (alcohol abuse, drugs addicted and their partner, migrants, female sex workers). Mothers of 22 babies (9.2\% of all women) abandoned maternity rights, in both CS and PT groups equal.

In addition to syphilis, $13.7 \%$ of pregnant women had concomitant STIs (gonorrhea, trichomoniasis, bacterial vaginosis, vulvovaginal candidiasis), in three women HIV infection and in one woman tuberculosis were found.

The partner notification was made only in 19 (18.6\%) pregnant women with active syphilis.

We found that $44.1 \%$ of pregnant women from the CS group did not visit the prenatal care facilities. In the PL group 34 women (43.6 \%) did not attend women's settings and respectively did not receive prenatal treatment. 
CS with symptoms was detected in the vast majority of infants - 85 (83.3\%), asymptomatic - only in $13(12.7 \%)$ cases.

Five babies, whose mothers were adequately treated before childbirth, were excluded from the study, as the diagnosis was not consistent with a global surveillance case definition [3].

In the meantime we had a limited data concerning 15 cases of stillbirth for the period since 2004 till 2006 years. Besides seven children (6.9\%) died at the age from 0.5 to 42 days from various severe complications of the CS on the background of multisystem organ failure, four of them - in the postneonatal period.

In the group of mothers, whose babies were diagnosed with CS, prenatal treatment was not carried out in 96 cases. Other six women of babies with CS had inadequate treatment including inproper regimen (4), serofast (1) and reinfection during pregnancy (1).

CS with symptoms was detected in the vast majority of newborn babies $-85(83.3 \%)$, asymptomatic - only in $13(12.7 \%)$ cases.

Infants from the CS group were born at gestational age of $35.6 \pm 0.3$ weeks, with the significant difference compared with the PL group $(\mathrm{p}<0,05)$.

Newborn babies were hospitalized with suspicion on CS in average very late ( $24.9 \pm 4.5$ day), and moreover their diagnostics and treatment began later.

In the neonatal period, the diagnosis of CS was established in $75(73.5 \%)$ newborns, and in $23(22.5 \%)$ - in post neonatal time, when the vast majority of infants develop symptoms of multisystem organ failure.

34 asymptomatic newborns (43.6 \%) out of 78 from PT group underwent treatment according to the scheme of the early CS, as born from mothers who did not get treatment of syphilis before childbirth, however, the diagnosis of the CS was not established to them.

\section{Discussion}

As our study showed, pregnant women with syphilis, according to socio-demographic characteristics, differ a little from uninfected pregnant women. They were young (average age at the time of delivery was 25.8 years), a significant number were unmarried (56.9\%), for most of them it was the first pregnancy (48\%). Therefore, most likely, these young women were infected with syphilis through unprotected sex with partners, including their husbands [8].

More than half of the women who gave birth to the infants with CS had a secondary (54.9\%) and high education (3.9\%), which means they were more accessible to preventive measures [9]. However, the percentage of those who had incomplete secondary education and less inclined to use preventive measures, reached almost $20 \%$.

Most women who have given birth to infants with CS belong to the active reproductive age, indicating the need for increased primary prevention of CS prevention among the female population [10]. The effectiveness of these measures will reduce the number of women with syphilis among the total number of patients and as a consequence - reduce the number of pregnant women with syphilis and, accordingly, reduce the number of newborns babies. In addition, our findings emphasize the need to increase the scope of primary prevention measures not only among the sexually active population as a whole, but also among adolescents, including those who are at risk group of infection, and can adopt behavior patterns associated with the risk of STI/HIV acquisition.

Women who were non-residents had less access to the free STI services. Besides, current requirements for confirmatory treponemal test, lead to a delay in the diagnosis and treatment of syphilis especially for women from risk group and rural areas [11]. The fact that in $81.4 \%$ of pregnant women with syphilis partner notification was not made indicates a significant risk of spreading of syphilis in the general population and passive surveillance [12].

At the time of maternity leave, the vast majority of women in all groups were unemployed or housewives. This fact indicates that the unemployed or housewives are at risk group for syphilis, as working women in order to receive maternity leave have to visit the prenatal care facilities regularly.

The vast majority of cases registered in Ukraine, according to the data of CMS, are symptomatic [13]. However, the possibility of conducting so called PT under the CS scheme for asymptomatic cases without appropriate notification creates the prerequisites for hiding some of the high 
suspected cases of the CS. According to the European guidelines in newborn babies that were born from mothers with untreated syphilis we should establish diagnosis of asymptomatic CS [14].

Analysis of the causes of CS shows that the main risk factors in Ukraine as in other Eastern European countries were the absence of prenatal care in $44 \%$ and treatment in $99 \%$ of pregnant women with syphilis [15].

In addition, the delay with prenatal diagnosis and prenatal treatment, late infection, quality of diagnostics, iatrogenic errors, refusal of women from current requirement for hospitalization, reinfection, birth at home can be attributed to risk factors for CS [16].

Since the ten years after our retrospective study, Ukraine closely approached the fulfillment of obligations to eliminate the CS, but still is facing challenges on case reporting, surveillance, monitoring and laboratory quality assurance.

Validation criteria with target indicators, which countries should meet regardless of their epidemiological characteristics, level of development or patterns of care for pregnant women, have been developed to confirm the EMTCT HIV infection and syphilis [17].

The minimum EMTCT for syphilis impact target is $\leq 50$ cases of CS per 100,000 live births.

Specific levels of service delivery also need to be met to accomplish EMTCT of syphilis. For validation of EMTCT of syphilis, the following process indicators and targets must be attained:

1. ANC coverage (at least 1 visit) of $\geq 95 \%$.

2. Coverage of syphilis testing of pregnant women of $\geq 95 \%$.

3. Treatment of syphilis-seropositive pregnant women of $\geq 95 \%$.

The analysis of the CMS data on the incidence of the CS in Ukraine since its peak in 1998 from 83 cases to isolated cases in recent years indicates the relevance of the country's indicators to the WHO indicator on the impact of EMTCT of syphilis.

However, our studies show that the statistical data on the CS does not reflect the real incidence.

In addition, three targets for EMTCT of syphilis were analyzed, and their relevance to WHO requirements.

In the CMS of the Ministry of Health available the data of pregnant women who are attending women' settings only in absolute numbers and we do not know real percent of the prenatal observation coverage.

According to the CMS, in Ukraine during the last decade, it is being testing more than $95 \%$ of pregnant women with syphilis and HIV (Table 1), which corresponds to the WHO target indicators for processes of validating EMTCT HIV and syphilis.

Table 1

Process indicators of EMTCT of HIV and syphilis in Ukraine

\begin{tabular}{lccccccc}
\hline & $\mathbf{2 0 1 0}$ & $\mathbf{2 0 1 1}$ & $\mathbf{2 0 1 2}$ & $\mathbf{2 0 1 3}$ & $\mathbf{2 0 1 4}$ & $\mathbf{2 0 1 5}$ & $\mathbf{2 0 1 6}$ \\
\hline $\begin{array}{c}\text { \% of pregnant women } \\
\text { tested on HIV }\end{array}$ & 96.9 & 99.2 & 99.2 & 99.3 & 99.2 & $\begin{array}{c}97.6 \text { (not enough } \\
\text { test systems) }\end{array}$ & 99.1 \\
$\begin{array}{c}\text { \% pregnant (new + those who } \\
\text { knew their HIV + status before } \\
\text { pregnancy) positive for HIV }\end{array}$ & 1.0 & 0.87 & 0.82 & 0.85 & 0.83 & 0.75 & 0.71 \\
$\quad \begin{array}{l}\text { \% of pregnant tested } \\
\quad \text { for syphilis }\end{array}$ & 96.24 & 96.00 & 96.60 & 96.57 & 96.98 & 97.77 & 97.32 \\
$\begin{array}{l}\text { \% pregnant positive for syphilis } \\
\text { Note: * without data from Crimea, Sevastopol since 2014, part of territory of anti-terrorist operation - from 2015 }\end{array}$ & 0.25 & 0.19 & 0.15 & 0.11 & 0.09 & 0.10
\end{tabular}

Comparison of prevalence rates for syphilis and HIV infection among pregnant women shows a smaller number of women tested for syphilis (Table 2).

This can be explained by the fact that the CMS receives data on the number of pregnant women tested for syphilis only up to 30 weeks of gestation, as opposed to HIV-infected, whose data include the results of rapid test during the childbirth [18]. 
Table 2

Prevalence rates for syphilis and HIV infection among pregnant women

\begin{tabular}{ccccccc}
\hline \multirow{2}{*}{ Years } & \multicolumn{2}{c}{$\begin{array}{c}\text { Prevalence rate of syphilis } \\
\text { number tested up to 30-week }\end{array}$} & \multicolumn{3}{c}{ Prevalence rate of HIV } \\
& positive number & \% & number of tested & positive (new) & \% \\
\hline 2007 & 449700 & 1305 & 0,29 & 598836 & 3085 & 0,52 \\
2008 & 477842 & 1380 & 0,29 & 643149 & 3563 & 0,55 \\
2009 & 486348 & 1502 & 0,31 & 585941 & 3234 & 0,55 \\
2010 & 474840 & 1235 & 0,26 & 579000 & 2797 & 0,48 \\
2011 & 475434 & 945 & 0,20 & 592836 & 2792 & 0,47 \\
2012 & 495405 & 778 & 0,16 & 585478 & 2633 & 0,45 \\
2013 & 477939 & 549 & 0,11 & 585544 & 2262 & 0,39 \\
$2014^{*}$ & 430012 & 382 & 0,09 & 522021 & 1872 & 0,36 \\
$2015^{*}$ & 388632 & 383 & 0,10 & 442766 & 1482 & 0,33 \\
$2016^{*}$ & 367776 & 365 & 0,10 & 412653 & 1314 & 0,32
\end{tabular}

Note: * - without data from Crimea, Sevastopol since 2014, part of territory of anti-terrorist operation - from 2015

Thus, due to the lack of serological data on syphilis late in pregnancy and during delivery, statistical data on the prevalence of syphilis among pregnant women do not cover women at risk group.

The CMS of the MHU of Ukraine does not provide data on the coverage of syphilis seropositive pregnant women with syphilis treatment, thus we have not official data about it. In addition, there is no guideline in Ukraine for the use of a single dose of intramuscular penicillin (benzathine penicillin) to prevent vertical transmission of syphilis in the seropositive pregnant women. In accordance with the current treatment protocols, penicillin benzathine is only allowed in the first half of pregnancy, but as our study showed in most cases multiple injections of the short-acting benzylpenicillin are prescribing to pregnant women. Moreover, in Ukraine, the practice of carrying out additional prophylactic treatment during pregnancy with a two-week course of benzylpenicillin during pregnancy remains, which is contrary to WHO and European recommendations [19].

It is a matter of a big public health concern that about $56 \%$ of pregnant women have been visited women' settings, but due to various reasons they have not been given a timely prenatal care and treatment until delivery [20].

\section{Conclusion}

Taking into account the high spreading of sexual transmission of HIV and numbers of pregnant women with HIV and reported MTCT in Ukraine crucial challenges are facing Ukraine on the way the to the elimination of the CS:

- new strategy on STI prevention with targets and targets indicators for CS;

- national protocols of the treatment of pregnant women should be adapted according to the European Guidelines;

- comprehensive data is required for surveillance and monitoring of CS elimination.

Given the policy of double elimination of MTCT of HIV and syphilis in Ukraine, the Maternity and Child Health and Prevention Mother-to-Child Transmission programs should be expanded with regard to universal access to syphilis diagnosis and treatment services for pregnant women at risk groups.

\section{References}

[1] Kiarie, J., Mishra, C. K., Temmerman, M., Newman, L. (2015). Accelerating the dual elimination of mother-to-child transmission of syphilis and HIV: Why now? International Journal of Gynecology \& Obstetrics, 130, S1-S3. Available at: https://www.ncbi.nlm.nih.gov/pubmed/26096725 doi: 10.1016/ j.ijgo.2015.05.002

[2] World Health Organization. Global Strategy for Elimination of Congenital Syphilis: Rationale and Strategy for Action (2007). Geneva: WHO, 48. Available at: http://www.who.int/reproductivehealth/publications/rtis/9789241595858/en/ 
[3] World Health Organization. Global Guidance on Criteria and Processes for Validation: Elimination of Mother-to-Child Transmission of HIV and Syphilis (2014). Geneva: WHO, 32. Available at: http:// www.who.int/reproductivehealth/publications/rtis/9789241505888/en/

[4] Investment case for eliminating mother-to-child transmission of syphilis: promoting better maternal and child health and stronger health systems (2012). Geneva: WHO, 30. Available at: http://www.who. int/reproductivehealth/publications/rtis/9789241504348/en/

[5] WHO validates countries' elimination of mother-to-child transmission of HIV and syphilis (2016). Availible at: http://www.who.int/mediacentre/news/statements/2016/mother-child-hiv-syphilis/en/

[6] About approving the clinical protocol providing medical care to children with suspicion of congenital syphilis (2009). Ministry of health protection of Ukraine, No. 769. Available at: http://consultant. parus.ua/?doc $=05 \mathrm{Y} 127 \mathrm{DC} 36$

[7] HIV infection in Ukraine (2017). Newsletter, 48. Kyiv: Tsentr hromadskoho zdorovia, 39. Available at: https://phc.org.ua/uploads/documents/c21991/be9c07b20fdcd077045761e95d0d82a6.pdf

[8] Ghebremichael, M. S., Finkelman, M. D. (2013). The Effect of Premarital Sex on Sexually Transmitted Infections (STIs) and High Risk Behaviors in Women. Journal of AIDS and HIV Research, 5 (2), 59-64. Available at: https://www.ncbi.nlm.nih.gov/pmc/articles/PMC3634578/

[9] Mirzaei, M., Ahmadi, K., Saadat, S., Ramezani, M. (2016). Instruments of High Risk Sexual Behavior Assessment: a Systematic Review. Materia Socio Medica, 28 (1), 46-50. doi: 10.5455/msm.2016.28.46-50

[10] Aral, S. O. (2004). Sexual risk behaviour and infection: epidemiological considerations. Sexually Transmitted Infections, 80, 8-12. Available at: http://sti.bmj.com/content/80/suppl_2/ii8 doi: 10.1136/ sti.2004.011866

[11] Luu, M., Ham, C., Kamb, M. L., Caffe, S., Hoover, K. W., Perez, F. (2015). Syphilis testing in antenatal care: Policies and practices among laboratories in the Americas. International Journal of Gynecology \& Obstetrics, 130, 37-42. doi: 10.1016/j.ijgo.2015.04.011

[12] Methods for surveillance and monitoring of congenital syphilis elimination within existing systems (2011). Geneva: WHO, 25. Available at: http://www.who.int/reproductivehealth/publications/ rtis/9789241503020/en/

[13] Center for Medical Statistics (CMS) of the Ministry of Health of Ukraine. Available at: http:// medstat.gov.ua/ukr/main.html

[14] Janier, M., Hegyi, V., Dupin, N., Unemo, M., Tiplica, G. S., Potocnik, M. et. al. (2014). 2014 European guideline on the management of syphilis. Journal of the European Academy of Dermatology and Venereology, 28 (12), 1581-1593. Available at: https://www.ncbi.nlm.nih.gov/pubmed/25348878 doi: 10.1111/ jdv.12734

[15] Herbert, L. J., Middleton, S. I. (2012). An estimate of syphilis incidence in Eastern Europe. Journal of Global Health, 2 (1). doi: 10.7189/jogh.01.010402

[16] Newman, L., Kamb, M., Hawkes, S., Gomez, G., Say, L., Seuc, A., Broutet, N. (2013). Global Estimates of Syphilis in Pregnancy and Associated Adverse Outcomes: Analysis of Multinational Antenatal Surveillance Data. PLoS Medicine, 10 (2), e1001396. doi: 10.1371/journal.pmed.1001396

[17] Taylor, M., Newman, L., Ishikawa, N., Laverty, M., Hayashi, C., Ghidinelli, M. et. al. (2017). Elimination of mother-to-child transmission of HIV and Syphilis (EMTCT): Process, progress, and program integration. PLOS Medicine, 14 (6), e1002329. doi: 10.1371/journal.pmed.1002329

[18] Morshed, M. G. (2014). Current trend on syphilis diagnosis: issues and challenges. Advances in Experimental Medicine and Biology, 808, 51-64. doi: 10.1007/978-81-322-1774-9_5

[19] WHO Regional technical consultation on monitoring progress and supporting capacity building to validate dual elimination of mother-to-child transmission of HIV and congenital syphilis (2015). Astana: WHO, 48. Available at: http://www.euro.who.int/_data/assets/pdf_file/0018/311706/Report-EMTCT-DraftFinal-040516.pdf

[20] Gomez, G. B., Kamb, M. L., Newman, L. M., Mark, J., Broutet, N., Hawkes, S. J. (2013). Untreated maternal syphilis and adverse outcomes of pregnancy: a systematic review and meta-analysis. Bulletin of the World Health Organization, 91 (3), 217-226. doi: 10.2471/blt.12.107623 